\title{
Structure Analysis of Human Prolidase Mutations gives insight into the Prolidase Deficiency disease mechanisms
}

\author{
$\underline{\text { P. Wilk }}^{1,2}$, M. Ühlein ${ }^{1}$, R. Piwowarczyk ${ }^{1}$, H. Dobbek ${ }^{2}$, M.S. Weiss ${ }^{1}$, U. Mueller ${ }^{1}$ \\ ${ }^{1}$ Helmholtz-Zentrum Berlin, Macromolecular Crystallography (HZB-MX), Elektronenspeicherring BESSY II, \\ Albert-Einstein-Str. 15, D-12489 Berlin, Germany. \\ ${ }^{2}$ Humboldt-Universität zu Berlin, Lebenswissenschaftliche Fakultät, Institut für Biologie \\ Strukturbiologie/Biochemie, Philippstraße 13, Leonor-Michaelis-Haus, D-10115 Berlin \\ piotrek.wilk@helmholtz-berlin.de
}

Prolidase is the only enzyme in humans capable of cleaving peptide bonds preceding proline or hydroxyproline in dipeptides. It is involved in the last steps of collagen catabolism and likely in the regulation of peptidic hormones. Diminished or absent prolidase activity was related to a rare autosomal disease referred to as Prolidase Deficiency (PD). Close to 20 different mutations related to this disorder were identified suggesting different mechanisms of loss of enzyme activity among affected individuals. To date, there is no definitive cure to PD. This is in part due to a limited understanding of the wild-type (wt) enzyme in terms of detailed substrate binding mode and as a consequence catalyzed reaction mechanism. Another reason might be the lack of structural information on the disease-related prolidase mutants. Here we describe the high resolution crystal structures of wt human prolidase in the ligand-free form and in the ligand-bound states. We present high resolution structure information on eight clinically relevant mutations introducing single amino acid changes into the prolidase chain. Based on this information we have identified different mechanisms of enzymes inactivation. Taken together, this information provides some insight into the pathologies related to this enzyme.

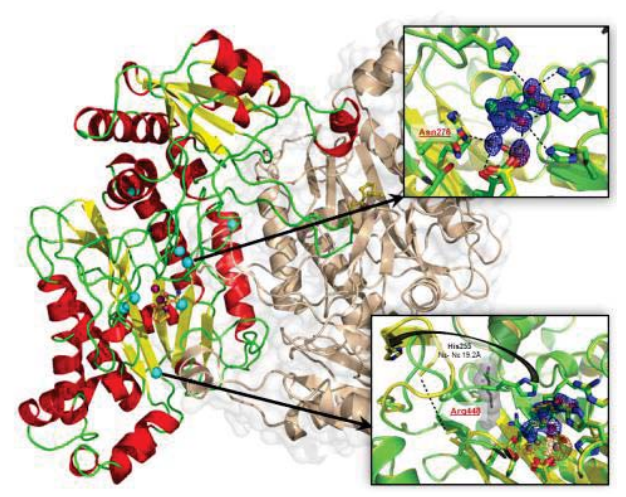

Figure 1: The overall structure of human prolidase is shown in cartoon representation with subunit $A$ colored according to secondary structure. The $\mathrm{Mn}^{2+}$ ions in the active site are shown as magenta spheres and GlyPro substrate as stick representation in orange. The positions of the mutated residues in chain $A$ are indicated by cyan spheres. The inlets show two examples of enzyme inactivation mechanism: (i) by losing one $\mathrm{Mn}^{2+}$ ion (Asp276Asn, upper panel) or (ii) by structure destabilization (Gly448Arg, lower panel). The structures of the mutants are superimposed onto the wt protein, 2Fo-Fc maps contoured at $2 \sigma$ are shown in blue and anomalous difference maps contoured at $10 \sigma$ in orange. 